\title{
QS能
}

Javier Yraola Burgos*

José Luis Briceño Romero**

\section{ESTRATEGIAS DE DESARROLLO ECONÓMICO DE MICHIGAN, CON ÉNFASIS EN VEHÍCULOS CONECTADOS Y AUTÓNOMOS}

Michigan constituye un ejemplo de cómo el establecimiento de objetivos estratégicos con políticas estables pueden transformar la economía de una región. Desde la última crisis su economía se ha diversificado, pero ha sido precisamente en el sector de automoción, el que ha tirado tradicionalmente de su economía, donde se están produciendo más cambios de interés. El sector de la movilidad se enfrenta a cambios profundos y Michigan ha preparado una estrategia para adaptarse a estos cambios, en particular en el segmento de vehículos conectados y autónomos. Además, el estado ha ampliado su estrategia a otros sectores con conexiones con la automoción, donde tiene fortalezas específicas. Como ejemplo de uno de ellos, se amplía el análisis de la estrategia de Michigan al sector de defensa y, dentro de esta, a la ciberseguridad.

Palabras clave: automoción, $\mathrm{I}+\mathrm{D}$, ciberseguridad, defensa.

Clasificación JEL: L62, L63, L86.

\section{Introducción}

Michigan, conocido como el estado de los Grandes Lagos, constituye un extraordinario ejemplo del dinamismo de la economía americana y de cómo el establecimiento de objetivos estratégicos a medio y largo plazo, con políticas estables y no ligadas al ciclo político, pueden transformar la economía de una región.

\footnotetext{
* Consejero de la Oficina Económica y Comercial de España en Chicago.

** Director de Departamento. Oficina Económica y Comercial de España en Chicago.

Versión de febrero de 2019.

DOI: https://doi.org/10.32796/bice.2019.3110.6792
}

Michigan, como estado claramente exportador respecto a la media nacional, está realizando en los últimos años un gran esfuerzo por diversificar su economía y hacerla más moderna y con menor dependencia de su gran industria tradicional, representada principalmente por los grandes fabricantes del sector de la automoción. En este sector, precisamente, su reto es ponerse a la vanguardia de la movilidad, para mantener su posición de liderazgo en el sector. Respecto a los datos de comercio bilateral con España, un dato muy significativo es el hecho de que España se situaba como el octavo país del que más importó Michigan en 2017 (aunque solo representara el 1\% del $\triangleright$ 
Javier Yraola Burgos y José Luis Briceño Romero

total de sus importaciones), con una subida de casi un 50\% respecto a 2016.

\subsection{Situación económica y clima de negocios en Michigan}

La economía de Michigan ha tenido históricamente un apoyo en la industria y, en particular, en el sector de automoción, muy superior al de la media nacional. Esta excesiva especialización sectorial, que lleva siempre aparejada una serie de riesgos asociados a los ciclos de expansión y contracción de dicho sector, generó en el caso de este estado un efecto arrastre sobre el conjunto de su economía en la última gran crisis económica. Michigan perdió cientos de miles de empleos industriales durante esa gran recesión, si bien también es cierto que fue el propio sector de automoción el que contribuyó en mayor medida a impulsar la extraordinaria recuperación económica de los últimos años.

Después del periodo más crítico sufrido por Michigan entre los años 2009 y 2010, la economía de este estado ha conseguido superar los niveles previos a la gran recesión. Su PIB era un $2 \%$ más alto en el año 2015 de lo que fue en 2006. Algo similar ocurrió con el empleo y con la renta per cápita durante esos años (Cuadro 1).
El estado ha diversificado su economía y ha reducido su nivel de desempleo de manera significativa en esta última fase de expansión económica hasta situarlo en un $4,3 \%$ en 2018 . La industria, aunque sigue jugando un papel primordial en esta economía, ha perdido cierto peso relativo, mientras que otros sectores como la sanidad o los servicios han crecido en mayor proporción. Desde que en el año 2006 se inició un proceso de diversificación de la economía de Michigan, sin tener en cuenta las Administraciones Públicas, la industria, que representaba el segundo gran sector en términos de trabajadores empleados, después de los de comercio, transporte y servicios públicos, perdió un puesto, según los datos del Michigan Bureau of Labor Market Information and Strategic Initiative. Desde 2016, la educación, los servicios sanitarios y los servicios profesionales han superado a la industria en número de empleados (Cuadro 2).

A pesar de que la industria sigue siendo un sector fundamental en este estado, la evolución y la transición experimentada por la industria de automoción hacia una mayor inversión en investigación y desarrollo en movilidad y en las nuevas tecnologías aplicadas a los vehículos han obrado este cambio. Como ha señalado Laurie Harbour (presidente y CEO de Southfield, empresa consultora en automoción): $D$

CUADRO 1

INDICADORES DE CLIMA DE NEGOCIOS DE MICHIGAN (2006-2015), AJUSTADO POR INFLACIÓN*

\begin{tabular}{|c|c|c|c|c|c|}
\hline & 2006 & 2010 & 2015 & $\begin{array}{c}\text { Cambio } \\
2015 \text { vs } 2006\end{array}$ & $\begin{array}{c}\text { Cambio } \\
2015 \text { vs } 2010\end{array}$ \\
\hline Población del estado & 10.036 .000 & 9.877 .000 & 9.923 .000 & $-1,10 \%$ & $0,50 \%$ \\
\hline PIB & $463.600 .000 \$$ & $415.500 .000 \$$ & $472.300 .000 \$$ & $1,90 \%$ & $13,70 \%$ \\
\hline Empleos & 4.326 .800 & 863.600 & 4.243 .700 & $-1,90 \%$ & $9,80 \%$ \\
\hline Tasa de paro & $7,00 \%$ & $12,60 \%$ & $5,40 \%$ & $-22,90 \%$ & $-57,10 \%$ \\
\hline Total estado renta per cápita & $395.000 .000 \$$ & $375.100 .000 \$$ & $424.800 .000 \$$ & $7,40 \%$ & $13,20 \%$ \\
\hline Renta per cápita & $39.426 \$$ & $37.990 \$$ & $42.812 \$$ & $8,60 \%$ & $12,70 \%$ \\
\hline
\end{tabular}


CUADRO 2

EMPLEO EN MICHIGAN POR SECTORES

\begin{tabular}{|c|c|c|c|}
\hline Sector & Empleos & Crecimiento 2016 (\%) & $\begin{array}{c}\text { Media crecimiento } \\
2012-2017(\%)\end{array}$ \\
\hline Servicios de hostelería y restauración & 377.661 & 1,3 & 2,1 \\
\hline Servicios y actividades de control de residuos & 287.315 & $-1,1$ & 0,8 \\
\hline Agricultura, forestal, pesca y caza & 31.934 & 2,4 & 4,1 \\
\hline Arte, entretenimiento y recreación & 53.645 & 4,0 & 2,8 \\
\hline Construcción & 159.155 & 2,8 & 4,5 \\
\hline Servicios educativos & 64.551 & $-0,9$ & 0,3 \\
\hline Finanzas y seguros & 147.793 & 1,7 & 1,7 \\
\hline Salud y asistencia social & 587.129 & 0,9 & 1,4 \\
\hline Información & 56.673 & $-0,7$ & 1,3 \\
\hline Gerencia de empresas y negocios & 64.908 & 6,3 & 3,5 \\
\hline Manufacturas & 610.635 & 1,8 & 2,6 \\
\hline Minería, canteras, extracción de petróleo y gas & 5.397 & $-1,0$ & $-3,1$ \\
\hline Otros servicios (excepto Admón. Pública) & 137.196 & 0,5 & 1,6 \\
\hline Servicios profesionales, científicos y técnicos & 292.899 & $-0,8$ & 3,5 \\
\hline Administración Pública & 555.731 & $-0,2$ & $-0,2$ \\
\hline Bienes raíces, alquileres y leasing & 53.817 & 2,5 & 2,3 \\
\hline Comercio minorista & 470.718 & $-0,3$ & 1,0 \\
\hline Transporte y almacenaje & 116.672 & 2,0 & 3,4 \\
\hline Empresas energéticas & 20.157 & 0,3 & 1,0 \\
\hline Comercio al por mayor & 172.156 & 0,2 & 1,6 \\
\hline
\end{tabular}

«es muy probable que los proveedores de automoción Tier 1 sean capaces de afrontar cualquier cambio en el modelo de negocio del sector porque se han especializado en nuevas tecnologías para vehículos».

Por otra parte, desde el punto de vista de la política económica, las medidas instrumentadas en los últimos años por el Gobierno de Michigan se han dirigido a mejorar el clima de negocios en el estado. En particular, se hizo énfasis en la reducción de impuestos, derogando el «Michigan Business Tax», un impuesto complejo con muchas excepciones, y sustituyéndolo por un nuevo impuesto con un tipo único de un $6 \%$, a la vez que se eliminaban exenciones y deducciones. Asimismo, se redujeron progresivamente los impuestos a la propiedad, en particular eliminando gradualmente los impuestos que gravaban la propiedad de determinados equipos productivos. Como resultado de estas reformas fiscales, Michigan tiene una presión fiscal inferior a la de la media nacional. El estado se situaba en el puesto 18.ㅇ en el ranking de estados con mayor carga fiscal de EE UU en 2010 y bajó al puesto 30. en solo cinco años. Otra medida que se aprobó fue una ley que hacía voluntario pagar cuotas a los sindicatos (Gráfico 1).

Por otra parte, en mayo de 2017 se creó una comisión ${ }^{1}$ para realizar un diagnóstico y establecer una serie de recomendaciones políticas, como estrategia a medio plazo, para garantizar la transformación y consolidación de la economía de Michigan. Recomendaciones que incluyen políticas en el ámbito de la educación, para reforzar la formación y consolidar y atraer $\triangleright$

\footnotetext{
$121^{\text {st }}$ Century Economy Commission, mayo de 2017.
} 


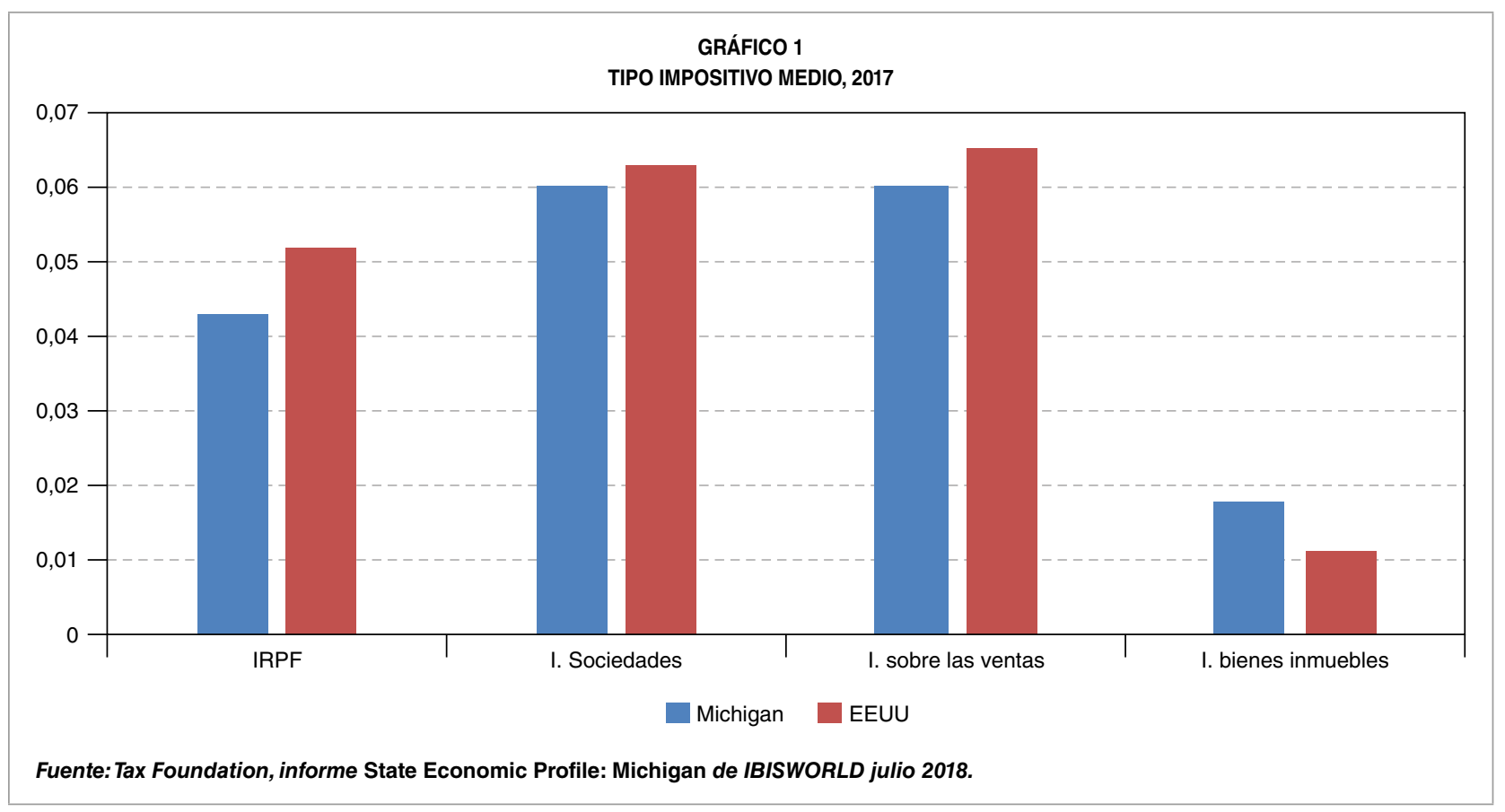

nuevo talento a la región, políticas de infraestructuras, de estabilidad fiscal y mejora del clima de negocios en general, etcétera.

\section{La estrategia de crecimiento de Michigan}

Como hemos visto, la salida de Michigan de la crisis económica de 2007 a 2009 tuvo que ver con una creciente diversificación de la economía del estado, en la que han aumentado los porcentajes de empleo en profesiones relacionadas con la salud y los servicios sociales, ciencia y tecnología. Asimismo el sector de automoción, impulsor tradicional de la economía del estado, también ha tenido un comportamiento excelente en los cinco años siguientes a la recesión, en los que se ha duplicado la producción de automóviles. Esto se corresponde con una recuperación notable en ventas a nivel nacional, que experimentaron un extraordinario periodo de siete años de crecimiento hasta llegar a máximos de récord en 2016, con
17,55 millones de vehículos. Complementando el aporte del sector de automoción, a estos avances se unió la mayor actividad industrial en el estado. De 2013 a 2017 subió el empleo en el sector industrial a una media anualizada de un 2,6\%. Esta subida es significativa, ya que contrasta con un entorno general de bajadas de empleo en la industria en EE UU por los fenómenos paralelos de deslocalización industrial y, sobre todo, por la automatización progresiva de la producción.

Parte de la explicación de esta subida son las medidas comentadas para hacer de Michigan un estado más atractivo para la inversión empresarial. El estado ha pasado a tener el octavo mejor puesto en impuestos a sociedades y el decimosegundo mejor en presión fiscal a empresas del país. Otras ventajas del estado son la alta formación de sus trabajadores y la especialización en sectores de alto potencial de crecimiento, como ingeniería, logística, defensa, educación superior, biociencias, recursos naturales y automoción. En este último sector, Michigan ha experimentado una notable $D$ 
transformación. Es conocido el fenómeno del desplazamiento del sector del automóvil desde el Medio Oeste, y Michigan, en concreto, hacia el sur de EE UU. Este movimiento fue impulsado por las primeras plantas que abrieron los fabricantes japoneses en los ochenta, atraídos por los menores costes laborales de la zona. Sin embargo, Michigan sigue produciendo más vehículos que ningún otro estado. En él están presentes 96 de las mayores 100 empresas en el sector, que representaban un $18,5 \%$ de la producción de EE UU en 2017. En cualquier caso, ese desplazamiento no alcanzó en igual medida a la I+D en el sector de automoción. Michigan ha mantenido su condición de líder en estas actividades, con una proporción de empleados que ha ido creciendo con el tiempo (Lavelle, 2018). En la actualidad, cuenta con 375 centros de I+D en automoción, de los cuales 120 son de empresas de matriz extranjera, o, para ilustrarlo de manera más gráfica, Michigan representa un $76 \%$ de la actividad de I+D del país en este campo. En los últimos cincuenta años se han concedido 20.000 patentes a empresas en el estado, tres veces más que al siguiente estado en el ranking.

\subsection{La posición de Michigan ante el futuro de la movilidad}

El sector de automoción se encuentra en mitad de un proceso de cambio profundo en el que hay múltiples tendencias disruptivas. La consultora McKinsey las agrupa en cuatro: movilidad diversificada, conducción autónoma, electrificación y conectividad. En las áreas donde más se ha avanzado en Michigan es en conducción autónoma y conectividad. Dado que estas tendencias se interrelacionan y alimentan entre sí, es difícil pronosticar cómo va a ser la industria de aquí a veinte años. Lo que sí se ha entendido desde Michigan es que esa evolución depende de conocimiento y talento, y que, por tanto, su condición de centro de investigación y desarrollo mundial en este campo le da una posición privilegiada en esta transformación. Esa visión parte del convencimiento de que no se puede parar el cambio tecnológi$\mathrm{co}$, por lo que se trata de convertirse en pionero o quedarse atrás, como indica esta frase de Kirk Steudle, director del Departamento de Transporte de Michigan durante trece años: «I have a firm belief that once something is technologically feasible, it is impossible to stop it ". (Slone, 2017). Y, efectivamente, el estado, en vez de obviar los cambios, se propuso abrir camino, legislando para permitir primero en 2013 testar vehículos autónomos y conectados (VAC 2 en lo sucesivo) en las carreteras del estado y luego en 2016 promulgando una serie de seis leyes con el objetivo de poner a Michigan a la vanguardia en este campo (MDOT, 2013). Entre esas leyes estaba la Public Act 332, que permitió la circulación de VAC sin necesidad de permisos o matrículas especiales y de camiones autónomos en convoy para obtener mayor eficiencia energética. Los esfuerzos de Michigan, por supuesto, no son los únicos, ya que hay 33 estados en EE UU que han adoptado legislación para VAC (National Conference of State Legislatures, 2018). El centro de investigación CAR (Center for Automotive Research), en Ann Arbor, ha realizado una comparativa de las áreas metropolitanas más avanzadas en VAC en el país y seleccionó las de Seattle, Pittsburgh, Detroit y San Francisco (San Francisco/San José y Oakland). Con respecto a estas dos últimas zonas, aunque Silicon Valley se ha $\triangleright$

2 CAV, por sus siglas en inglés, que corresponden a Connected and Autonomous Vehicles. 
visto tradicionalmente como el epicentro de los cambios que se están sucediendo en automoción, con el tiempo se observa una evolución más hacia colaboración entre estos dos polos de desarrollo que hacia competencia, por el alto grado de complementariedad entre las dos. Así, hay un número creciente de alianzas o integración por compra de empresas tecnológicas y empresas de automoción ${ }^{3}$. Si bien se ha producido una implantación progresiva de las últimas en las ciudades de la bahía de San Francisco, también se produce el fenómeno a la inversa, cada vez con más empresas de tecnología y grupos de inversión implantándose en Michigan. Uber ha abierto un centro tecnológico de VAC en el suburbio de Wixom, y el último ejemplo es el anuncio de Waymo, la spin off de Alphabet (Google) para vehículos autónomos, que construirá con Magna la primera planta enteramente dedicada a este tipo de automóviles en el sudeste de Michigan.

Las ventajas que atraen a esas empresas hacia Michigan y, en particular, hacia los condados en el sudeste del estado en torno a Detroit, son su gran especialización productiva, la alta densidad de centros de I+D y diseño de automoción en general y específicamente de VAC, y las instalaciones y pistas de pruebas existentes para estos vehículos. Algunos ejemplos de esto último son MCity, en la Universidad de Michigan, o el American Center for Mobility (ACM), en Ypsilanti. MCity es una iniciativa público-privada en la que participan 65 empresas y el Departamento de Transporte de Michigan. Se trata

\footnotetext{
3 Alianza de Waymo con FCA: www.businessinsider.com/waymo-fcastrengthen-self-driving-partnership-2018-5

Acuerdos de Uber con Toyota y GM con Lyft: www.fredbeans.com/ toyota-partners-with-uber-gm-with-lyft/

Compra de GM de la empresa Cruise Automation: http://fortune. com/2016/03/11/gm-buying-self-driving-tech-startup-for-more-than-1billion/

Compra de Ford de la empresa Argo Al: https://techcrunch.com/ 2017/02/18/ford-invests-in-pittsburgh-based-argo-ai-to-build-selfdriving-cars-by-2021/
}

de un espacio que simula un entorno urbano con infraestructura de tráfico y en el que se prueban tecnologías de comunicación entre vehículos e infraestructura (V2I, por sus siglas en inglés) y entre vehículos (V2V). El American Center for Mobility (ACM) abrió sus puertas en abril de 2018 y cuenta con un moderno circuito de pruebas, verificación y certificación de VAC. Otros puntos fuertes del área metropolitana de Detroit son las veinte universidades de la zona que ofrecen titulaciones de ingeniería. El estado cuenta con 117.000 ingenieros en total y ostenta un liderazgo nacional en la proporción de ingenieros industriales y mecánicos. La Universidad de Michigan es una institución de referencia en investigación sobre VAC. Su Instituto de Investigación de Transporte (UMTRI, por sus siglas en inglés) es una pieza clave en proyectos piloto de seguridad de vehículos y en la gestión del centro MCity mencionado anteriormente. En Michigan también se puede encontrar un entramado institucional que incluye organismos específicamente desarrollados para trabajar en el progreso de la movilidad del futuro y en una coordinación adecuada entre los sectores público y privado y las universidades. La creación de PlanetM, una alianza pública-privada dentro de la agencia de inversiones estatal, la Michigan Economic Development Corporation (MEDC), se ha configurado como dinamizadora de las actividades en el sector y punto de conexión entre los distintos actores del sistema ${ }^{4}$.

Otros grupos de trabajo y organismos activos en este campo son: - Michigan Council of Future Mobility (Dpto. de transporte): https:// www.michigan.gov/whitmer/0,9309,7-387-90501_90626-405828--, 00.html

- Michigan State University - Mobility: https://mobility.msu.edu

- CAV Working Group at CAR (Center for Automotive, Research) (Dpto. de transporte): https://www.cargroup.org/cav-workinggroup/

- Connected Vehicle Trade Association: http://www.connected vehicle.org/

- MichAuto - Detroit Regional Chamber: http://www.detroitchamber. com/econdev/chamber-initiatives/michauto-universal-name/

- Mobile Technology Association of Michigan: http://www.gomobilemichigan.org/ 


\title{
Ejemplos de casos piloto en VAC en Michigan
}

\author{
Magna y May Mobility
}

Magna International, la empresa canadiense de componentes del automóvil, y May Mobility, una startup de Ann Arbor, han colaborado para desarrollar una flota de seis miniautobuses autónomos para la empresa Bedrock que, a partir de junio de 2018, desplaza a trabajadores desde un parking hasta las oficinas de la empresa en Detroit.

\section{Continental AG y Magna International}

Otro ejemplo reciente de tecnologías de movilidad autónoma lo tenemos en el acuerdo internacional entre las empresas Continental AG $^{5}$ (empresa alemana con gran presencia en EE UU) y Magna International Inc, con respaldo público. En agosto de 2017, ambas compañías realizaron una prueba conjunta de conducción autónoma de vehículos que cubrieron una distancia de $\mathbf{3 0 0}$ millas cruzando dos pasos de frontera a través de un túnel entre Michigan (EE UU) y Canadá. Este ensayo es el fruto de un acuerdo de colaboración transfronteriza entre PlanetM, el Departamento de Transporte del estado de Michigan (MDOT) y el Ministerio de Transporte de Ontario (OMT).

\section{NAVYA Autonomous bus}

Otro de los proyectos piloto que está funcionando con mayor éxito es el caso de un minibús eléctrico sin conductor fabricado por la empresa francesa NAVYA en pruebas en MCity desde junio de 2018. Este minibús tiene capacidad para transportar a quince pasajeros y utiliza sensores LiDAR y un GPS para navegar de manera autónoma.

Además del vehículo localizado en las instalaciones de pruebas de la Universidad, hay otros dos vehículos en uso que forman parte del proyecto. Estos dos minibuses transportan estudiantes, profesores y empleados en el campus norte de dicha universidad. Se trata del primer proyecto de vehículo sin conductor en EE UU centrado en la investigación del comportamiento de los consumidores y la recolección de estos datos. NAVYA abrió una planta de ensamblaje de sus vehículos al oeste de Detroit en octubre de 2018.

\section{TARDEC, vehículo autónomo}

En octubre de 2017 el centro del ejército americano conocido como TARDEC (US Army Tank Automotive Research, Development and Engineering Center), que desarrolla tecnologías avanzadas en vehículos terrestres militares, realizó una demostración de su última tecnología durante un test de conducción entre Port Huron (Michigan) y Sarnia (Ontario, Canadá), cruzando en dos ocasiones el puente Blue Water entre los dos países. Se trataba de un convoy de vehículos que incluía dos tractores M915 equipados con el sistema de movilidad autónoma «TARDEC Autonomous Mobility Appliqué Systems». Esta tecnología incorpora un sistema de alerta al conductor y de conducción semiautónoma que permite conducir el vehículo a través de un recorrido programado previamente. La prueba incluyó asimismo una tecnología que permite que el vehículo que lidera el convoy dé instrucciones automáticas a los demás vehículos que le siguen en términos de aceleración, frenada o detención simultanea de todo el grupo. Este sistema permite que un convoy circule con los vehículos casi pegados y actúe como si fuera un solo vehículo o un tren.

5 La empresa alemana Continental AG inició sus pruebas con vehículos con diferentes niveles de autonomía en Nevada en 2012 y ya han completado más de 15.000 millas de pruebas sin accidentes. 
Hay que tener en cuenta que Michigan también tiene debilidades, según su propia evaluación (Brugeman, Fiorelli, Spulber, 2017): por un lado está la falta de suficientes profesionales que combinen el conocimiento del mundo del automóvil con las tecnologías de la información; sin embargo, ese es un problema compartido con otros estados, por tratarse de un campo emergente. Otro punto en el que Michigan debe mejorar es en aumentar el número de grupos de inversión y capital riesgo y la cuantía de las inversiones, ya que está por detrás de Silicon Valley en este aspecto. Por la gran cantidad de centros de I+D en el estado, falta cerrar la brecha de comercialización de tecnologías, o sea la diferencia entre los dólares de I+D invertidos y la inversión correspondiente de capital riesgo. No obstante, este factor puede considerarse un atributo positivo a la hora de atraer inversores, ya que representa oportunidades sin explotar.

\section{Otros sectores}

\subsection{Defensa y ciberseguridad}

Muchas de las mismas ventajas que operan para el sector de automoción, como la alta proporción de ingenieros en el estado o el peso de la fabricación avanzada y el número de centros en $I+D$, también hacen que el estado sea interesante para la industria de Defensa. Michigan cuenta con 4.000 empresas y 100.000 empleos que dependen del sector, que aporta $9.720 \mathrm{mi}-$ llones de dólares a la economía del estado. Una institución importante para Michigan es el US Army Detroit Arsenal, el centro de mando para tanques, vehículos acorazados y armamentos (TACOM, por sus siglas en inglés). Esta unidad gestiona las compras, el inventario y la gestión del $65 \%$ del material y los armamentos para el Ejército, así como la logística y el despliegue de dicho material. Cuenta con muchos programas de vital importancia, como el centro de ingeniería e I+D para tanques y vehículos acorazados (TARDEC, por sus siglas en inglés; ver caso piloto). La presencia de estos centros redunda en más oportunidades de contratos para las empresas locales y de acuerdos de colaboración con universidades y centros de investigación del estado. Además de este centro, en Michigan hay dos bases aéreas de la Guardia Nacional (Selfridge y Battle Creek), tres centros de entrenamiento (Camp Grayling, Alpena y Fort Custer) y la Agencia de Logística de Defensa.

Por ese motivo, Michigan se ha esforzado en desarrollar una estrategia para el sector. El gobernador Snyder impulsó en 2015 el plan estratégico denominado «Protect and Grow» para ampliar la ya considerable presencia de empresas de Michigan como proveedores del Ejército, proteger las instalaciones militares en Michigan y expandir las misiones encomendadas a las bases del Ejército en el estado. En el plan colaboraron representantes políticos, la industria de defensa, militares, agencias de desarrollo económico y entidades académicas de las diferentes regiones de Michigan. Lideró el esfuerzo el Michigan Defense Center, que depende de MEDC $^{6}$. Entre otros puntos, el plan enfatiza la necesidad de promover y salvaguardar las capacidades de compras, tecnología y logística en el Detroit Arsenal, fortalecer y promover los vínculos entre la defensa y el automóvil doméstico y liderar el país en la integración de operaciones cibernéticas del Departamento de Defensa y el estado. Una de las recomendaciones implementadas ha sido el lanzamiento de un $\triangleright$

\footnotetext{
6 Michigan Economic Development Corporation: www.michiganbusiness.org

Michigan Defense Center: www.arsenalofinnovation.com
} 
portal llamado Bid Targeting System (BTS) ${ }^{7}$ exclusivo para las empresas de Michigan que las ayuda a buscar oportunidades y asesora para maximizar sus posibilidades de conseguir adjudicarse licitaciones de seguridad y defensa.

Mención específica merece el sector de ciberseguridad, un área en la que Michigan ha desarrollado un gran esfuerzo y una política decidida para situarse entre los estados pioneros en el país ${ }^{8}$. El estado tiene una red de entidades y unos procedimientos muy desarrollados para prevención, tratamiento y respuesta a incidentes relacionados con la ciberseguridad ${ }^{9}$. Desde 2007 cuenta con un centro de operaciones de inteligencia que reúne y disemina información y alertas de amenazas de ciberseguridad. En 2011, el gobernador Snyder lanzó un primer plan de acción en ciberseguridad que tuvo continuidad con un segundo plan en 2015. Los objetivos eran mejorar la preparación del estado y aprovechar las oportunidades económicas que ofrecía el sector ${ }^{10}$.

En julio de 2013 se creó un centro de mando único para seguridad cibernética ${ }^{11}$ en la Policía Estatal para centralizar las acciones de respuesta y coordinación de todas las entidades en casos de incidentes graves ${ }^{12}$. Se completó esta estructura con un cuerpo civil de ciberseguridad $^{13}$, con expertos que, de forma voluntaria, están preparados para colaborar con las entidades del estado para resolver incidentes en caso de que se declare un estado de emergencia. Otra iniciativa del estado fue desarrollar

\footnotetext{
7 http://www.bidtarget.org

8 https://pellcenter.org/wp-content/uploads/2017/02/State-of-theStates-Report.pdf

9 https://www.us-cert.gov/sites/default/files/c3vp/s/tt/Michigan Cyber_Governance_Case_Study_508.pdf

10 Michigan announces Cyber Initiative: www.michigan.gov/som/ 0,4669,7-192-29701-263758--,00.html

Michigan Cyber Initiative 2015: https://www.michigan.gov/documents/cybersecurity/Mich_Cyber_Initiative_11.13_2PM_web_474127_7.pdf

11 Michigan Cyber Command Center (MC3).

12 Michigan Intelligence Operations Center (MioC).

13 Michigan Cyber Civilian Corps (MiC3).
}

un programa de educación y entrenamiento específico para ciberseguridad en colaboración con la Guardia Nacional y el sector privado, el Michigan Cyber Range. Su objetivo es formar de manera novedosa y práctica a alumnos, profesionales y entidades públicas conforme al marco que establece NICE (National Initiative for Cybersecurity Education) ${ }^{14}$. Por su conexión con los automóviles conectados y autónomos, la ciberseguridad aplicada a la automoción es, naturalmente, una de las prioridades del estado. Un factor que ha contribuido a que Michigan destaque en este campo es la tradición de las empresas de automoción de colaborar entre ellas, favoreciendo que haya grupos de trabajo y una comunidad muy activa en la que se comparten esfuerzos de investigación y desarro$11 \mathrm{o}^{15}$ y se avance más rápidamente.

\section{Conclusiones}

En Michigan encontramos el ejemplo de un estado que se ha visto forzado, por las circunstancias económicas, a reflexionar profundamente sobre sus fortalezas y debilidades y la manera de afrontar un cambio tecnológico que afecta a la principal industria del estado: la automoción. Michigan cuenta con instituciones sólidas y muy volcadas a favorecer los negocios empresariales y fomentar la cooperación entre el sector público y el privado. Han detectado las áreas en las que la industria se transformaba $y$, en vez de ir a remolque de los $\triangleright$

14 El marco educativo NICE (National Initiative for Cybersecurity Education) está dirigido por The National Institute of Standards and Technology (NIST), una entidad del Departamento de Comercio, trabajando en colaboración con entidades académicas y el sector privado para fijar las categorías y los léxicos comunes en el trabajo de ciberseguridad para uso educativo y privado.

15 A través de asociaciones como Automotive Industry Action Group (www.aiag.org/), Auto Alliance (https://autoalliance.org/) y Auto-ISAC (www.automotiveisac.com), entre otras. 
cambios, han intentado ponerse a la vanguardia de los mismos. En estos momentos de profunda transformación tecnológica resulta más difícil que nunca hacer predicciones de futuro, pero la estrategia del estado de enfocarse en la educación de su fuerza laboral, sus esfuerzos en la atracción y retención de talento y en I+D, parece que está dando sus frutos en términos de crecimiento económico y de atracción de inversión extranjera, por el alto ritmo de anuncios de plantas de producción que se han sucedido en los últimos años. Entre ellos, podemos destacar un grupo de empresas españolas de componentes de automoción que tienen su sede en el estado, como Grupo Antolín y Corporación Gestamp. Gestamp amplió sus dos plantas en el estado en 2016 y abrió un centro de I+D en $2017^{16}$. Grupo Antolín inauguró una nueva planta en junio de $2018^{17}$. CIE Automotive también apostó por el estado adquiriendo la división de techos de la empresa Inteva en septiembre de 2018. Ficosa también tiene su sede en Michigan, así como otras empresas del sector, como Zanini, Teknia, Canela y Cumsa. Las empresas españolas están plenamente implicadas en los organismos y grupos de trabajo que diseñan el futuro de la movilidad en Michigan.

\section{Bibliografía}

[1] Austin, J., Good, J., y Kolluri, A. (2017). Jobs, Michigan \& Leadership in the economy of tomorrow. Recuperado de https://static1.squarespace.com/static/5a31a397e5dd5b97586824 e5/t/5bbd4f2f9140b76c6c5bb9f5/1539133257 475/Jobs+Michigan+and+Leadership+in+the + Economy+of+Tomorrow.pdf

\footnotetext{
16 www.gestamp.com/prensa/comunicados-de-prensa. aspx?NewID=3320

17 www.grupoantolin.com/en/grupo-antolin-opens-new-factory-usa
}

[2] Baruah, S. et al. (2017). Building Michigan's. $21^{\text {st }}$ Century Economy. Recuperado de https:// www.michiganbusiness.org/4aec0b/globalassets/documents/reports/medc-reports/21c_ economy_commission_final_report.pdf

[3] Biggs, J. (2017, febrero). Ford invests $\$ 1$ billion in Pittsburgh-based Argo Al to build self-driving cars by 2021. Techcrunch. Recuperado de https://techcrunch.com/2017/02/18/fordinvests-in-pittsburgh-based-argo-ai-to-buildself-driving-cars-by-2021/

[4] Business Leaders for Michigan (2017). Plan for a stronger Michigan. Recuperado de https:// businessleadersformichigan.com/wp-content/ uploads/2018/03/Plan-for-a-Stronger-Michiganbooklet-022618-1.pdf

[5] Center for Automotive Research (2017). Strategic Growth Plan for Connected and Automated Vehicle Assets in Southeast Michigan. Recuperado de https://www.cargroup.org/wpcontent/uploads/2017/08/CAR-CAV-RegionalPlan-Final-12-October-2017.pdf

[6] Debord, M. (2018, mayo). Waymo and FiatChrysler are bulking up their self-driving partnership. Recuperado de https://www.businessinsider.com/waymo-fca-strengthen-selfdriving-partnership-2018-5

[7] Deloitte (2019). Express lane ahead. Finding new roads to value creation as the automotive industry transforms. Recuperado de https:// www2.deloitte.com/content/dam/insights/us/ articles/4899_Automotive-news-supplement/ DI_Automotive-News-supplement.pdf

[8] Fred Beans (2018). Toyota partners with Uber, GM with Lyft. Recuperado de https://www.fredbeans.com/toyota-partners-with-uber-gmwith-lyft/

[9] Gao, P., Kaas, H., Mohr, D., y Wee, D. (2016). Disruptive trends that will transform the auto industry. Mckinsey. Recuperado de https:// www.mckinsey.com/industries/automotive-andassembly/our-insights/disruptive-trends-thatwill-transform-the-auto-industry

[10] Ibisworld (2018, julio). State Economic Profile. Michigan. Recuperado de https://www.ibisworld.com/industry-insider/media/1785/michiganeconomicprofile.pdf

[11] Korosec, K., y Primack, D. (2016, marzo). GM Buying Self-Driving Tech Startup for More Than $\$ 1$ Billion. Fortune. Recuperado de $\triangleright$ 
http://fortune.com/2016/03/11/gm-buying-selfdriving-tech-startup-for-more-than-1-billion/

[12] Lavelle, M. (2018, agosto). Michigan's Populated Regions Driving the State's Unemployment Rate Lower. Michigan Economy. Recuperado de http://michiganeconomy.chicagofedblogs. org/?cat=3

[13] Michigan Bureau of Labor Market Information and Strategic Initiatives (2018, julio). Michigan's Hot 50 Careers through 2026. Recuperado de http://milmi.org/Portals/198/publications/ Hot_50_Brochure_2026.pdf?ver $=2018-10-30-084757-123$

[14] Michigan Council on Future Mobility (2018). Annual Report 2018. Recuperado de https:// planetm.michiganbusiness.org/globalassets/ pdf/mcf_mobility_report.pdf

[15] Michigan Department of Transportation (2013). Public Act 231 of 2013; Section 665(3), Testing and Operation of Automated Vehicles. Recuperado de https://www.michigan.gov/documents/mdot/PA_231_of_2013_Section_6653 _AV_legislation_report_512858_7.pdf

[16] Michigan Department of Transportation (2017). 2017 Connected and Automated Vehicle strategic plan. Recuperado en https://www.michigan.gov/documents/mdot/MDOT_CAV_ Strategic_Plan_FINAL_623811_7.pdf

[17] Michigan Economic Development Corporation (2015). Michigan's Economic Comeback.

[18] Michigan Economic Development Corporation (2017). Michigan is Auto Mobility. Recuperado en https://future.michigan.gov/download/ww3szqmi/application\%2Fpdf

[19] National Conference of State Legislatures (2018, julio).Autonomous vehicles. Self-driving vehicles enacted legislation. Recuperado de http://www.ncsl.org/research/transportation/ autonomous-vehicles-self-driving-vehiclesenacted-legislation.aspx

[20] Senate Fiscal Agency (2018, noviembre). Michigan Economic Indicators. Recuperado de
http://www.senate.michigan.gov/sfa/Publications/EconInd/MEI_MostRecent.pdf

[21] Slone, S. (2017, julio). Michigan Department of Transportation Director Kirk Steudle: States Should "Get Out of the Way» on Autonomous Vehicle Policy. Recuperado de http://knowledgecenter.csg.org/kc/content/michigandepartment-transportation-director-kirksteudle-states-should-get-out-way

[22] State of Michigan (2014). Michigan Cyber Initiative 2015. Leading the Nation: An interagency, public-private collaboration. Recuperado de https://www.michigan.gov/documents/ cybersecurity/Mich_Cyber_Initiative_11.13_ 2PM_web_474127_7.pdf

[23] Sumagaysay, L. (2019, enero). Waymo Plans to Build 'World's First' AV Factory in Michigan. The San Jose Mercury News. Recuperado de http://www.govtech.com/fs/automation/ Waymo-Plans-to-Build-Worlds-First-AVFactory-in-Michigan.html

[24] The Michigan Defense Center (2016). Protect and Grow: A Strategic Plan for Michigan's Defense and Homeland Security Economy. Recuperado de https://www.michigan.gov/documents/snyder/Michigan Protect and $_{-} \mathrm{Grow}_{-} \mathrm{Public_{- }}$ Report_2016_525922_7.pdf

[25] University of Michigan Transportation Research Institute (Umtri) \& Workforce Intelligence Network for Southeast Michigan (WIN) (2017). Connected and automated vehicles skills gap analysis. Recuperado de https:// winintelligence.org/wp-content/uploads/ 2017/03/WIN_ATLAS-UMTRI_Reportsinglepages-rs.pdf

[26] Wilkinson, M. (2018, marzo). Michigan's economy is bigger than ever. Now is the time to diversify. Bridge Magazine. Recuperado de https://www.bridgemi.com/economy/ michigans-economy-bigger-ever-now-time-diversify 